\title{
Immunophenotyping of Clinically Isolated Syndrome Patients Who Did or Did Not Convert to Multiple Sclerosis
}

\section{Maryam Nakhaei-Nejad}

Division of Neurology, Department of Medicine, University of Alberta

\section{Chieh-Hsin Lee}

Division of Neurology, Department of Medicine, University of Alberta https://orcid.org/0000-0003-0253296X

\section{David Barilla}

Division of Neurology, Department of Medicine, University of Alberta

\section{Carlos Camara-Lemarroy}

Department of Clinical Neurosciences, University of Calgary

\section{Bei Jiang}

Mathematical and Statistical Sciences, University of Alberta

\section{Wee Yong}

Department of Clinical Neurosciences, University of Calgary

Fabrizio Giuliani ( $\nabla$ giuliani@ualberta.ca )

https://orcid.org/0000-0002-4042-8533

\section{Research}

Keywords: Multiple sclerosis, Immunophenotyping, Peripheral blood mononuclear cells

Posted Date: July 15th, 2020

DOI: https://doi.org/10.21203/rs.3.rs-41481/v1

License: (c) (1) This work is licensed under a Creative Commons Attribution 4.0 International License. Read Full License 


\section{Abstract}

\section{Background}

Clinically isolated syndrome (CIS) is the prodromal phase of multiple sclerosis (MS) disease course, with patients having experienced one neurological episode.

Up to $90 \%$ of CIS patients develop subsequent MS, so it is important to differentiate those who will and will not convert to Relapsing Remitting Multiple Sclerosis (RRMS) in the future, which will allow for earlier treatment. We aimed to test whether peripheral blood immunophenotype could predict conversion from clinically isolated syndrome (CIS) to multiple sclerosis (MS).

Methods

Multicolor flow cytometry was used to identify up to 50 peripheral blood mononuclear cell (PBMC) subpopulations in fresh blood samples. Further, cryopreserved PBMCs processed in another center were identically immunophenotyped in the same facility where the fresh blood specimens were analysed.

Results

In addition to major subpopulations ( $T$ and B lymphocytes, monocytes, dendritic cells (DC) and natural killer (NK) cells), we measured the frequencies of multiple $\mathrm{CD} 4^{+}$and $\mathrm{CD} 8^{+} \mathrm{T}$ cell, monocyte, and $\mathrm{NK}$ subsets. Among the studied subpopulations, DCs were significantly lower in CIS who converted to MS (CIS-C) compared to patients who did not (CIS-N). Also, CD56 CD $16^{+}$atypical NK subset was lower in CISC. We also studied cryopreserved CIS patient samples from a previous clinical trial involving the CIS populations. We observed significant differences between fresh and cryopreserved PBMC subpopulations. We were not able to detect the same differences in the cryopreserved samples.

Conclusions

Our data using fresh blood samples reveals that while there were significant differences between CIS-N and CIS-C in the DCs and in one of the NK subsets, there was no overall difference between the immunophenotypes of CIS patients who did and did not convert to MS. These changes could be of physiological relevance to the disease pathogenesis. The fact that these differences were not observed in cryopreserved samples could mean that using fresh blood from patients may provide a more accurate tool to study the immune system composition.

\section{Background}

Multiple sclerosis (MS) is a chronic inflammatory demyelinating disease of the central nervous system and is a common cause of disability in younger adults, especially women (1). 
One major characteristic of the disease is the invasion of proinflammatory lymphocytes through the blood-brain barrier (BBB) into the central nervous system (CNS). There, these cells are thought to contribute to the demyelination, oligodendrocyte and neuronal death, and axonal loss (1).

Clinically isolated syndrome (CIS) is the prodromal phase of MS defined by patients having experienced their first episode of neurological symptoms. Out of those patients, up to $90 \%$ are subsequently diagnosed with MS within 2 years $(2,3)$. Early identification of patients who would develop MS is of significant clinical importance since it has been shown that early immunomodulatory treatment can improve the outcome of the disease $(4,5)$.

Several factors have been proposed as predictors for conversion of CIS to MS, such as number and location of lesions in the CNS $(6,7)$, presence of oligoclonal bands (OCB) in the cerebrospinal fluid (CSF) (8), and immune system composition $(9,10)$. CNS imaging and CSF analysis are routinely used in clinical practice to identify the risk of CIS conversion to MS (2). However, due to the invasive nature of CSF collection, the identification of peripheral blood biomarkers has great practical value.

The composition of the immune system in CIS patients has been compared to healthy individuals or MS patients in several studies $(11,12)$. One of these studies showed a lower proportion of a regulatory $T$ cell (Treg) $\left(C D 45 R A^{+} F_{0 x P}{ }^{l o}\right.$ ) and follicular $T$ regulatory cell subsets $(\mathrm{Tfr})$, in recently diagnosed untreated CIS patients when compared to healthy controls. In addition, all Treg and Tfr subsets showed a downregulation of the Helios transcription factor, which is involved in functional stability of Tregs. Overall, the authors provided evidence for a disturbed balance between suppressive and proinflammatory Treg and Tfr subpopulations in CIS (11). Arellano et al. analyzed plasma levels of several cytokines in CIS and in different clinical forms of MS (12). In CIS patients, the researchers observed higher levels of IFN- $\gamma$ and IFN-y /IL-10 ratio when compared to relapsing remitting MS (RRMS) patients. In addition, RRMS patients had a higher T helper 17 (Th17) frequency while CIS patients had a higher percentage of $\mathrm{T}$ helper 1 (Th1) than Th17 (12). Certain B cell subsets have also been proposed as predictors of conversion to MS. Villar et al. detected a higher percentage of $\mathrm{CD} 5^{+} \mathrm{B}$ cells in CIS patients who converted to MS (9). Aktura et al. observed that patients who converted to MS had lower $\mathrm{CD} 19^{+} \mathrm{B}$ cells but higher frequency of unswitched memory B cells (CD27 ${ }^{+}$IgD ${ }^{+}$cells) (13).

In our study, we aimed to identify whether the peripheral blood immunophenotype could predict conversion to MS. We characterized the peripheral blood mononuclear cell (PBMC) of $40 \mathrm{CIS}$ patients that were followed up to three years. Samples were collected in two centres, with one of the centres providing PBMCs that were cryopreserved for storage. In addition to deep cell surface marker analysis and comparison of patients who did or did not convert to MS, we were able to compare the effect of cryopreservation on various immune subsets. Our data indicate that some lymphocyte subpopulations were different between CIS that convert to MS versus non-converters and that several subsets were significantly affected by cryopreservation.

\section{Methods}




\section{Patients}

Fresh blood samples from a total of 19 patients with a diagnosis of CIS were collected from the Kaye Edmonton Clinic, Edmonton, Alberta Canada after approval from the University of Alberta Ethics Committee. These patients had regular clinical follow-up once a year and MRI brain scans were performed every 6 months to monitor disease activity and dissemination in time. Conversion to MS was based on the revised McDonald's criteria of 2010 (14). MS was diagnosed with one relapse due to evidence of dissemination in space and time by new clinical symptoms and/or evidence of new lesions on the MRI. Informed consent was obtained before the blood draw to study human PBMC in accordance to Ethics Committee recommendations, with the median time between onset and sample collection being 504 days. Whole blood was used for flow cytometry as described below.

For the cryopreserved sample, under an approved protocol (University of Calgary IRB), blood was drawn from 21 participants in a clinical trial involving the use of minocycline in patients with CIS (15). Conversion to MS was based on the McDonald's criteria of 2005 (16). PBMCs were purified using FicollPlaque $^{\text {TM }}$ PLUS (GE Healthcare) centrifugation. Heparinized blood was diluted 1:1 with phosphatebuffered saline (PBS) and carefully layered over Ficoll and centrifuged at $870 \mathrm{xg}$ for 30 minutes. The mononuclear cell layer was washed with PBS and centrifuged for 10 minutes at $390 \mathrm{xg}$. The supernatant was then resuspended in $20-30 \mathrm{ml}$ of PBS containing $1 \%$ heat-inactivated human serum (where cell density was determined) and centrifuged for 10 minutes at $170 \mathrm{xg}$ and pellets were resuspended to a final density of $40 \times 10^{6} \mathrm{cells} / \mathrm{ml}$ in $100 \%$ human serum, and an equal volume of $20 \%$ DMSO in human serum was added for a final density of $20 \times 10^{6} \mathrm{cells} / \mathrm{ml}$. Tubes were then transferred to a Cryofreezing pack (containing isopentane) and then stored until analysis in liquid nitrogen. Cryopreserved PBMC samples were shipped to our centre, where the samples were thawed and resuspended in RPMI medium supplemented with $10 \%$ heat inactivated FBS, $1 \%$ antibiotics and $1 \%$ Glutamine and let recover overnight on ultra-low adherent plates to prevent monocyte adhesion to dishes. To prevent cell-clumping $20 \mathrm{U}$ of DNase was added to the medium (all reagents from Gibco, Thermo Fisher). The next day, cell surface marker staining was done with a volume equal to $1 \times 10^{6}$ cells $/ \mathrm{ml}$ of PBMCs (described below).

\section{Flow Cytometry}

All antibodies and reagents were purchased from BD Biosciences (Mississauga, ON). For freshly derived sample analysis, $100 \mu \mathrm{l}$ of blood collected in sodium heparin Vacutainers was mixed with freshly made antibody cocktails diluted in Brilliant stain buffer for each panel. Antibodies' clone and product numbers are as described in our previous paper (17). Red blood cells were then lysed using 1XPharmlyse (BD FACS $^{\text {TM }}$ lysing solution) according to instruction manual, no later than 6 hours from the time of collection (stored at room temperature). Considerations for panels designs were described previously (17). Data was analysed using FlowJo (Version 10.3. FlowJo, LLC, 2006-2017). Results are expressed as a percentage of child to parent gating or as \% of total cell count over the original PBMC gate. Gating strategies for each panel have been used as previously described (17).

\section{Statistical Analysis}


All statistical analysis was done using R (18). Student's t-test was used for individual comparisons between groups. A p-value of $<0.05$ was considered statistically significant. For the overall comparison of immunophenotypes between the groups, the partial least square discriminant analysis (PLS-DA) function in the mixOmics $\mathrm{R}$ package was used (19). The optimal number of components, error rate, and area under the curve (AUC) were assessed via the perf function in the package using 5 -fold cross-validation repeated 10 times.

\section{Results}

To gain more insights into the immunophenotype of CIS patients, we characterized the expression of up to 23 surface receptors in the mononuclear cell population by designing 2 multiparametric flow cytometry panels (17). These panels allow us to detect major populations of immune cells including lymphocytes ( $T$ cells and B cells), natural killer (NK) cells, monocytes, and dendritic cells (DCs) as well as their respective subpopulations. Table 1 indicates the demographic of patients who were enrolled in this study. All samples were taken while patients were diagnosed with CIS. We characterized the immunophenotype of CIS patients who did (CIS-C) or did not (CIS-N) progress to MS within the follow-up period (3 years post sample collection). For the donors fresh blood samples, conversion to MS was based on the McDonald's criteria 2010 (14). Upon receiving blood samples, fresh blood was immunophenotyped by flow cytometry as described in the Methods section. Since lymphocytes are considered major players in the MS pathogenesis, we compared the frequencies of lymphocyte populations between CIS patients who did or did not convert to MS. No significant difference was observed in the overall PBMC population $(p=0.48$, data not shown), $C D 3^{+} \mathrm{T}$ cell (Fig. $\left.1 \mathrm{~A}\right)$ and $\mathrm{CD} 4^{+}$or $\mathrm{CD} 8^{+} \mathrm{T}$ cell subpopulations (Fig. 1B) or their ratio $(\mathrm{p}=$ 0.64 , data not shown) between patients who converted or not to MS. Next, we looked at the expression of receptors, CCR7 and CD45RA, to identify the rate of naïve, effector, effector memory and central memory cells within $\mathrm{CD}^{+}$or $\mathrm{CD} 8^{+} \mathrm{T}$ cell subpopulations. There was no significant difference between these subsets in converted versus non-converted patients (Fig. 1C-D). Next, we analyzed CD $4^{+}$cells for their expression of chemokine receptors that characterize $\mathrm{CD} 4^{+}$cells into $\mathrm{T}$ helper (Th) subsets. We did not detect any significant differences in frequencies of Th1, Th2, Th17, Th22, or Tfh levels between converted and non-converted patients (Fig. 1E). 
Table 1

Demographics of patients with CIS who either did (CIS-C) and or did not (CIS-N) convert to MS who were recruited for the purpose of immunophenotyping their peripheral blood mononuclear cells.

\begin{tabular}{|lllll|}
\hline & \multicolumn{2}{l}{ Fresh Blood } & \multicolumn{2}{l|}{ Cryopreserved } \\
\hline & CIS-C & CIS-N & CIS-C & CIS-N \\
\hline Age, y, mean \pm SD & $40 \pm 9$ & $39 \pm 13$ & $31 \pm 8$ & $33 \pm 7$ \\
Females, \% & 71 & 58 & 57 & 86 \\
\hline
\end{tabular}

We sought to identify differences in levels of $\mathrm{CD}^{1} 9^{+} \mathrm{B}$ cells or its various subtypes between CIS-C and CIS-N patients. Although there was a trend towards lower B cell counts in CIS-C compared to CIS-N, this was not statistically significant (Fig. 2A). Further, there were no changes in naïve and various memory subsets of the $\mathrm{CD} 19^{+}$cells (Fig. 2B).

Circulating monocytes, NK cells and DC have been shown to play a role in MS disease activity. The overall population of $\mathrm{CD} 14^{+}$monocytes, as well as their classical $\left(\mathrm{CD} 16^{-}\right)$and non-classical $\left(\mathrm{CD} 16^{+}\right)$ subsets were not different between CIS-C and CIS-N (Fig. 3A-B). Interestingly, patients who converted to MS had a lower number of DC (gated CD3-CD19- ${ }^{-}$D 14- ${ }^{-} \mathrm{HLA}-\mathrm{Dr}{ }^{+}$) compared to non-converted CIS (Fig. 3C). Patients who converted to MS also had higher levels of a subset of NK cells, CD 56 ${ }^{\text {Dim }}$ CD $16^{+}$, while the overall population of NK cells was not different among the two groups of patients (Fig. 3D).

The data from the individual subpopulations were confirmed by the analysis of the overall difference between CIS-C and CIS-N in fresh blood samples that showed a separation with an AUC of 0.426 and an error rate of 0.437 , indicating no significant difference in their immune profiles (Fig. 4A).

Next, we aimed to characterize the samples from the cryopreserved PBMCs. These PBMCs were allowed to recover overnight in ultra-low attachment dishes before flow cytometry analysis. It has been previously shown that cryopreservation can differentially affect various PBMC subtypes $(20,21)$. We compared the frequency of all the subpopulations between fresh or cryopreserved samples. Among the major PBMC populations $\mathrm{CD} 8^{+} \mathrm{T}$ cells were significantly different between the freshly processed samples and the cryopreserved ones. In addition, some lymphocyte subsets had significantly different frequencies between fresh and cryopreserved samples (Fig. 4B). Also, no CD14 ${ }^{+}$cells were detected in the cryopreserved PBMCs. Analysis of the difference between cryopreserved and fresh blood samples showed a separation with an AUC of 1 and an error rate of 0 , indicating that cryopreservation leads to a significant overall change in the PBMC subpopulations (Fig. 4C). Thus, we characterized the immunophenotype of converted and non-converted CIS patients separately in fresh blood and cryopreserved samples. No differences in the frequency of major populations or their subsets were detected between converted and non-converted in the cryopreserved patient samples (data not shown). 
PLS-DA analysis of the CIS-C and CIS-N difference in the overall immune profile within cryopreserved PBMC samples indicated a separation with an AUC of 0.382 and an error rate of 0.481 , indicating no significant difference in the immune profiles of CIS patients who converted to MS or not (Fig. 4D).

\section{Discussion}

In this study, we aimed to identify differences in the peripheral blood immune cell subpopulations between CIS patients who did and did not convert to MS following the revised McDonald criteria of 2005 and 2010. Several studies have pointed to the importance of the immune system in the CIS pathogenesis and progression to $M S(9,10,12,22,23)$. Rinaldi et al. characterized the immunophenotype of $20 \mathrm{CIS}$ patients for one year, categorizing patients according to their MRI activity (10). Approximately $10 \%$ of the analyzed immune subsets were found to separate patients according to their lesion numbers, indicating the importance of the peripheral blood immunophenotype in the disease process (10). Posova, et al. studied 180 patients that were treated with interferon $\beta 1$ a to identify disability and relapse progression (22). They reported $\mathrm{CD} 4^{+}$naïve lymphocytes as a possible predictor of disease activity (22). In our untreated patient population, we were not able to detect any significant changes in the proportion of major $\mathrm{CD}^{+} \mathrm{T}$ cell subpopulations nor $\mathrm{CD}^{+}$and $\mathrm{CD} 8^{+}$subsets including the proportion of $\mathrm{T}$ helper cells and their naïve/memory phenotype.

In a study by Aktura et al., B cells were examined in less than 10 patients and lower CD $19^{+} \mathrm{B}$ cell frequency was observed in CIS patients that converted to MS as well as a higher unswitched memory $B$ cell proportion (23). Although we saw the same trend in B cell frequencies in our study, this difference did not reach statistical significance. In addition, we did not detect any significant differences in the unswitched memory subset in converted patients.

In one study, CIS patients with higher disease activity were reported to have higher $\mathrm{CD} 56^{+} \mathrm{CD} 16^{+} \mathrm{NK}$ cells, suggesting the involvement of NK cells in the pathogenesis of the disease (10). Although we did not detect any change in the that subset, CIS-C patients had a lower percentage in the $\mathrm{CD}^{2} 6^{-} \mathrm{CD} 16^{+} \mathrm{NK}$ cell subpopulation compared to CIS-N. Expansion of this NK subset has been reported in HIV-infected patients and in individuals with post-traumatic stress disorder $(24,25)$. This subpopulation has been shown to secrete lower amounts of cytokines and express lower cytolytic activity (24). The importance of this NK subtype in autoimmunity has not been studied and warrants further investigations.

Additionally, we observed a lower frequency of dendritic cells in the CIS-C patient population. Linking innate and adaptive immune system, DCs are involved in a number of immune system functions such as tolerance and development of effector and regulatory Th cells $(26,27)$. Indeed, chronic progressive MS patients have lower plasmacytoid DCs compared to RRMS and healthy controls (28). This suggests that the lower frequency of $\mathrm{DC}$ we observe in CIS-C could be of pathophysiological relevance.

Our results suggest that the cryopreservation of PBMCs can lead to significant changes in both specific subpopulations and the overall immunophenotype, as shown in Fig. 4C. We found significant differences 
in multiple CD4 + T cell, B cell, and NK cell subpopulations, along with significantly higher percentage of $\mathrm{CD}^{+} \mathrm{T}$ cells in fresh blood samples. Our findings are in disagreement with previous studies regarding the effect of cryopreservation on immune cell populations. In contrast to our results, Mata, Mahmood (29) showed no significant change in $\mathrm{CD} 16^{+} \mathrm{CD} 56^{\mathrm{Dim}}$ and monocyte subpopulations, both of which are significantly decreased in our samples. Others have shown no changes in the major PBMC subpopulations (i.e. $\mathrm{CD} 4^{+}$and $\mathrm{CD} 8^{+} \mathrm{T}$ cells, $\mathrm{B}$ cells, and $\mathrm{CD} 3^{-} \mathrm{CD} 19^{-}$cells) (30); while our results agree with most of their findings, we found a significant decrease in the percentage of $\operatorname{CD} 8^{+} \mathrm{T}$ cells following cryopreservation. These changes have implications into the design of studies that rely on the use of cryopreservation, including clinical trials. The immune cell subpopulations detected in cryopreserved samples may not be representative of the actual frequencies found in fresh blood. Cryopreserved samples may be validly used as biomarkers to differentiate between groups or for diagnoses as long as the results are only compared to other cryopreserved samples, preferably with the same freeze/recovery processes. These samples should not be used to determine effects of interventions or treatments, as it would be difficult to separate the effect of the treatment from that of cryopreservation. Future studies should examine the effect of cryopreservation on samples from the same individuals, as our study compared separate groups of patients. The effect of different methods on cryopreservation and recovery should also be examined to establish whether there are optimal methods to minimize the effect the processes have on the cell populations.

\section{Conclusion}

Our study shows that using multicolor flow cytometry analysis, the overall immunophenotype of CIS patients who convert to MS following two different sets of criteria (McDonald 2005 and 2010) is not significantly different from those patients who do not convert. These findings raise the possibility that factors responsible for conversion from CIS to MS could be dependent on the CNS compartment. Our results also showed significant differences in the immune subpopulations between fresh blood and cryopreserved samples, suggesting that these differences need to be taken into account when designing studies that require an accurate map of the peripheral immunophenotype.

\section{List Of Abbreviations}

CIS, clinically isolated syndrome; MS, multiple sclerosis; PBMC, peripheral blood mononuclear cell; DC, dendritic cell; NK, natural killer; BBB, blood-brain barrier; CNS, central nervous system; Treg, regulatory $T$ cell; Tfr, follicular T regulatory cell; RRMS, relapsing remitting MS; Th17, T helper 17; Th1, T helper 1; PLSDA, partial least square discriminant analysis ; AUC, area under the curve; Th, T helper

\section{Declarations}

\section{Ethics approval and consent to participate}


Fresh blood samples were collected following approval from the University of Alberta Ethics Committee. For the cryopreserved sample, the protocol was approved by the University of Calgary IRB.

\section{Consent for publication}

Notapplicable

\section{Availability of data and materials}

The datasets used and/or analysed during the current study are available from the corresponding author on reasonable request.

\section{Competing interests}

$\mathrm{MNN}, \mathrm{CHL}, \mathrm{DB}, \mathrm{BJ}$, and VWY report no disclosures.

CML has received personal compensation for consulting, serving on a scientific advisory board, speaking, or other activities with EMD Serono, Inc.

FG served on the scientific advisory board of Alberta MS Network; received speaker honoraria from Merck Serono, Biogen, Genzyme, and Roche; consulted for Merck Serono, Biogen, Genzyme, and Roche; and received research support from Biogen Canada and Alberta Ministry of Innovation and Advanced Education.

\section{Funding}

This study was sponsored by Biogen Canada Inc, Government of Alberta, and the MS Society of Canada. The funding bodies had no role in the analysis and interpretation of the data nor in the writing of the manuscript.

\section{Authors' contributions}

MNN designed and conceptualized study, interpreted the data, and drafted the manuscript for intellectual content. $\mathrm{CHL}$ analyzed the data, interpreted the data, and drafted the manuscript for intellectual content. DB collected samples and analyzed the data. CCL provided feedback for the design and conceptualization of study, collected samples, and revised the manuscript for intellectual content. BJ designed statistical analysis tools and provided feedback for the manuscript for intellectual content. VWY provided feedback for the design and conceptualization of study, collected samples, and revised the manuscript for intellectual content. FG designed and conceptualized study, collected samples, interpreted the data, and revised the manuscript for intellectual content. All authors read and approved the final manuscript.

\section{Acknowledgements}

Notapplicable 


\section{References}

1. Dendrou CA, Fugger L, Friese MA. Immunopathology of multiple sclerosis. Nature reviews Immunology. 2015;15(9):545-58.

2. Miller DH, Chard DT, Ciccarelli O. Clinically isolated syndromes. Lancet Neurol. 2012;11(2):157-69.

3. Metz LM. Clinically Isolated Syndrome and Early Relapsing Multiple Sclerosis. Continuum (Minneap Minn). 2019;25(3):670-88.

4. Thrower BW. Clinically isolated syndromes - Predicting and delaying multiple sclerosis. Neurology. 2007;68:12-S5.

5. La Mantia L, Di Pietrantonj C, Rovaris M, Rigon G, Frau S, Berardo F, et al. Interferons-beta versus glatiramer acetate for relapsing-remitting multiple sclerosis. Cochrane Database of Systematic Reviews. 2016(11).

6. Tintoré M, Rovira A, Rio J, Nos C, Grive E, Tellez N, et al. Baseline MRI predicts future attacks and disability in clinically isolated syndromes. Neurology. 2006;67(6):968-72.

7. Tintore M, Rovira A, Arrambide G, Mitjana R, Rio J, Auger C, et al. Brainstem lesions in clinically isolated syndromes. Neurology. 2010;75(21):1933-8.

8. Tintore M, Rovira A, Rio J, Tur C, Pelayo R, Nos C, et al. Do oligoclonal bands add information to MRI in first attacks of multiple sclerosis? Neurology. 2008;70(13):1079-83.

9. Villar LM, Espino M, Roldan E, Marin N, Costa-Frossard L, Muriel A, et al. Increased peripheral blood CD5 + B cells predict earlier conversion to MS in high-risk clinically isolated syndromes. Multiple Sclerosis Journal. 2011;17(6):690-4.

10. Rinaldi L, Gallo P, Calabrese M, Ranzato F, Luise D, Colavito D, et al. Longitudinal analysis of immune cell phenotypes in early stage multiple sclerosis: distinctive patterns characterize MRI-active patients. Brain. 2006;129:1993-2007.

11. Jones AP, Trend S, Byrne SN, Fabis-Pedrini MJ, Geldenhuys S, Nolan D, et al. Altered regulatory T-cell fractions and Helios expression in clinically isolated syndrome: clues to the development of multiple sclerosis. Clin Transl Immunol. 2017;6.

12. Arellano G, Acuna E, Reyes LI, Ottum PA, De Sarno P, Villarroel L, et al. Th1 and Th17 Cells and Associated Cytokines Discriminate among Clinically Isolated Syndrome and Multiple Sclerosis Phenotypes. Frontiers in Immunology. 2017;8.

13. Aktura SD, Yilmaz V, Ozkan-Yasargun D, Ulusoy C, Tuzun E, Turkoglu R. Peripheral blood memory B cell frequency predicts conversion from clinically isolated syndrome to multiple sclerosis. Multiple Sclerosis Related Disorders. 2018;23:9-14.

14. Polman CH, Reingold SC, Banwell B, Clanet M, Cohen JA, Filippi M, et al. Diagnostic Criteria for Multiple Sclerosis: 2010 Revisions to the McDonald Criteria. Ann Neurol. 2011;69(2):292-302.

15. Metz LM, Li DKB, Traboulsee AL, Duquette P, Eliasziw M, Cerchiaro G, et al. Trial of Minocycline in a Clinically Isolated Syndrome of Multiple Sclerosis. New Engl J Med. 2017;376(22):2122-33. 
16. Polman $\mathrm{CH}$, Reingold SC, Edan G, Filippi M, Hartung H-P, Kappos L, et al. Diagnostic criteria for multiple sclerosis: 2005 revisions to the “McDonald Criteria”. Ann Neurol. 2005;58(6):840-6.

17. Nakhaei-Nejad M, Barilla D, Lee C-H, Blevins G, Giuliani F. Characterization of lymphopenia in patients with MS treated with dimethyl fumarate and fingolimod. Neurology - Neuroimmunology Neuroinflammation. 2018;5(2).

18. R Core Team. R: A language and environment for statistical computing. Austria: R Foundation for Statistical Computing. Vienna; 2018.

19. Rohart F, Gautier B, Singh A, Lê Cao K-A, mixOmics. An R package for 'omics feature selection and multiple data integration. PLOS Computational Biology. 2017;13(11):e1005752.

20. Kotsakis A, Harasymczuk M, Schilling B, Georgoulias V, Argiris A, Whiteside TL. Myeloid-derived suppressor cell measurements in fresh and cryopreserved blood samples. J Immunol Methods. 2012;381(1):14-22.

21. Zhang W, Nilles TL, Johnson JR, Margolick JB. The effect of cellular isolation and cryopreservation on the expression of markers identifying subsets of regulatory T cells. J Immunol Methods. 2016;431:31-7.

22. Posova H, Horakova D, Capek V, Uher T, Hruskova Z, Havrdova E. Peripheral blood lymphocytes immunophenotyping predicts disease activity in clinically isolated syndrome patients. BMC Neurol. 2017;17.

23. Aktura ŞD, Yılmaz V, Özkan-Yaşargün D, Ulusoy C, Tüzün E, Türkoğlu R. Peripheral blood memory B cell frequency predicts conversion from clinically isolated syndrome to multiple sclerosis. Multiple Sclerosis Related Disorders. 2018;23:9-14.

24. Mavilio D, Lombardo G, Benjamin J, Kim D, Follman D, Marcenaro E, et al. Characterization of CD56(-)/CD16(+) natural killer (NK) cells: A highly dysfunctional NK subset expanded in HIV-infected viremic individuals. Proc Natl Acad Sci USA. 2005;102(8):2886-91.

25. Bersani FS, Wolkowitz OM, Milush JM, Sinclair E, Eppling L, Aschbacher K, et al. A population of atypical CD56(-)CD16(+) natural killer cells is expanded in PTSD and is associated with symptom severity. Brain Behav Immun. 2016;56:264-70.

26. de Jong EC, Smits HH, Kapsenberg ML. Dendritic cell-mediated T cell polarization. Springer Semin Immun. 2005;26(3):289-307.

27. Cools N, Ponsaerts P, Van Tendeloo VFI, Berneman ZN. Balancing between immunity and tolerance: An interplay between dendritic cells, regulatory T cells, and effector T cells. J Leukocyte Biol. 2007;82(6):1365-74.

28. Thewissen K, Nuyts AH, Deckx N, Van Wijmeersch B, Nagels G, D'hooghe M, et al. Circulating dendritic cells of multiple sclerosis patients are proinflammatory and their frequency is correlated with MS-associated genetic risk factors. Multiple Sclerosis Journal. 2014;20(5):548-57.

29. Mata MM, Mahmood F, Sowell RT, Baum LL. Effects of cryopreservation on effector cells for antibody dependent cell-mediated cytotoxicity (ADCC) and natural killer (NK) cell activity in $51 \mathrm{Cr}$ release and CD107a assays. J Immunol Methods. 2014;406:1-9. 
30. Perdomo-Celis F, Salgado DM, Castañeda DM, Narváez CF. Viability and Functionality of Cryopreserved Peripheral Blood Mononuclear Cells in Pediatric Dengue. Clin Vaccine Immunol. 2016;23(5):417-26.

\section{Figures}

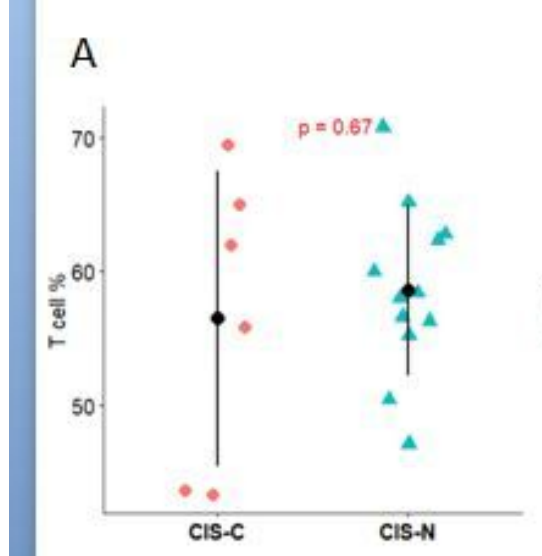

D

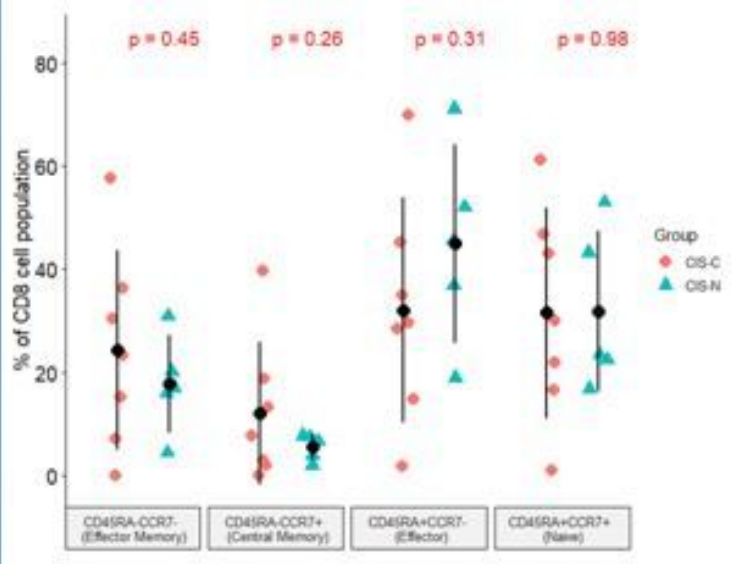

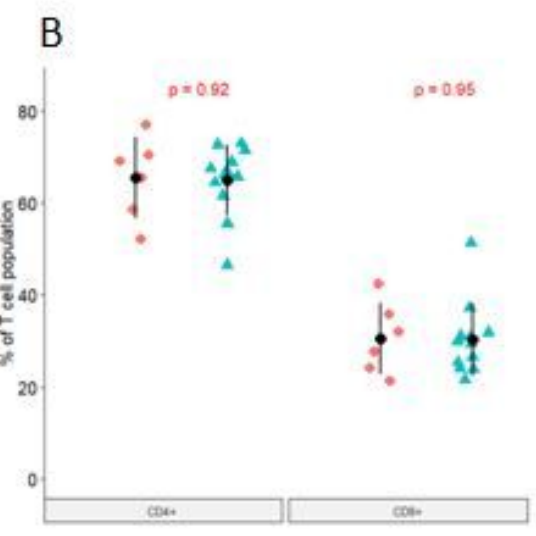
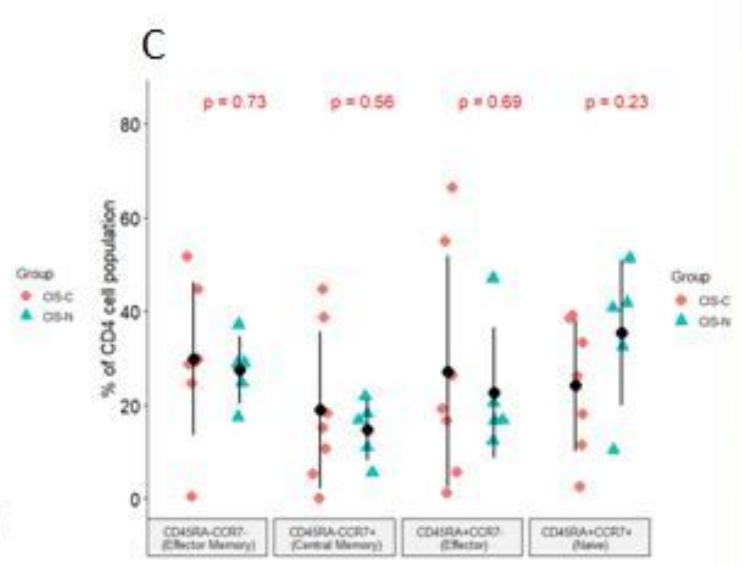

$\mathrm{E}$

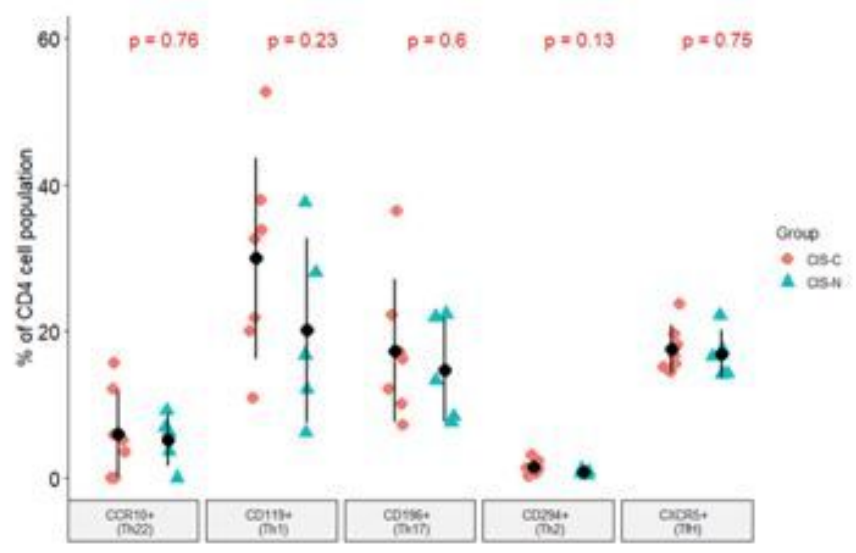

Figure 1

Overall $\mathrm{T}$ cell frequency and $\mathrm{CD} 4+$ and $\mathrm{CD} 8+\mathrm{T}$ cell subsets Flow cytometry analysis of fresh peripheral blood samples of patients who converted to MS (CIS-C, n=7) and those who did not (CIS-N, n=12) examining the abundance of $C D 3+(A), C D 4+$ and $C D 8+(B), C D 4+$ memory populations, CD 8+ memory populations (D), and T helper cells (E). The frequency of cell populations are as a $\%$ of their parent population as indicated, with T cells being as a \% of PBMCs. The black dot indicates the mean of each group with the vertical line being the SD. The p-values of the comparisons are as shown in the insets. 
A

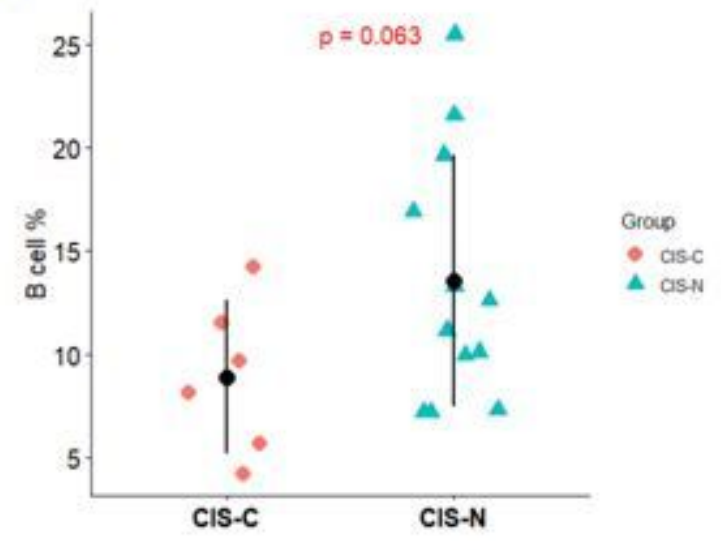

B

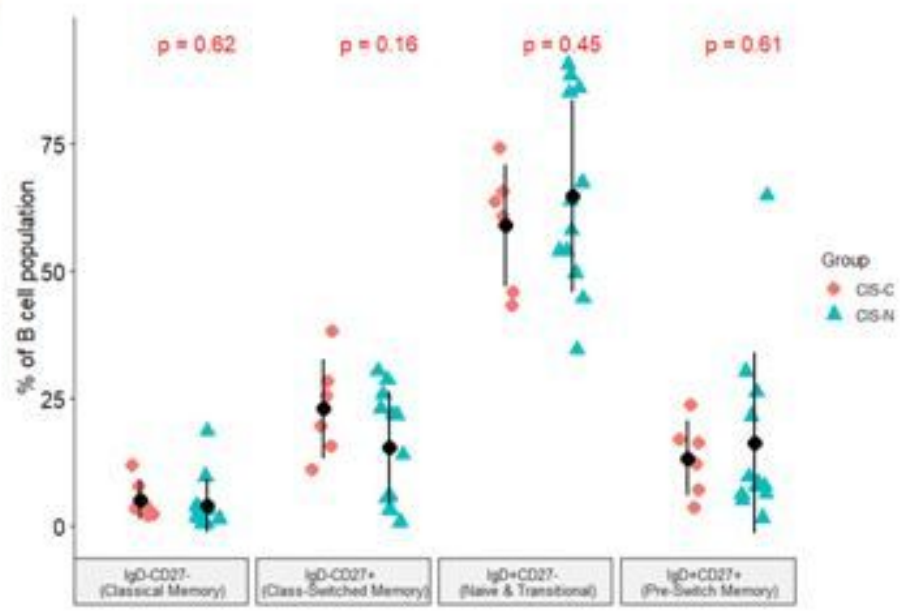

\section{Figure 2}

Overall B cell amounts and B cell subsets Flow cytometry analysis of fresh peripheral blood samples of patients who converted to MS (CIS-C, n=7) and those who did not ( $\mathrm{CIS}-\mathrm{N}, \mathrm{n}=12$ ) examining the abundance of $C D 19+(A)$, and $B$ cell subsets $(B)$. The frequency of cell populations are as a $\%$ of their parent population as indicated, with $B$ cells being as a \% of PBMCs. The black dot indicates the mean of each group with the vertical line being the SD. The p-values of the comparisons are as shown in the insets. 
A
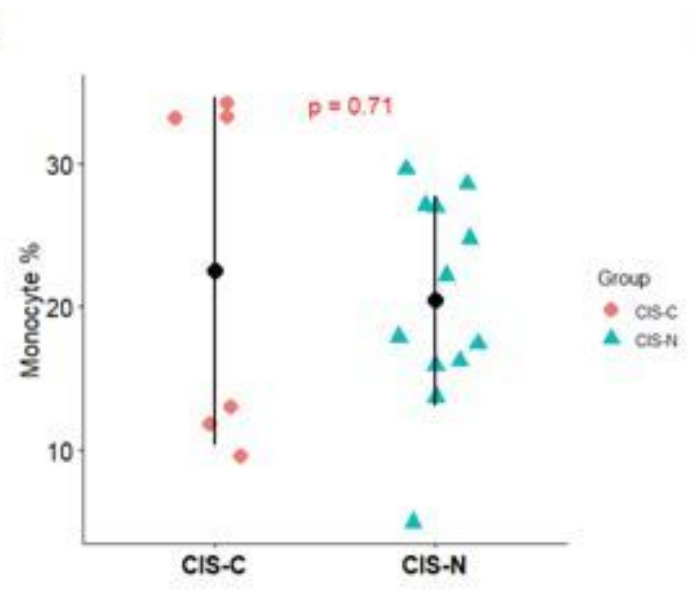

C

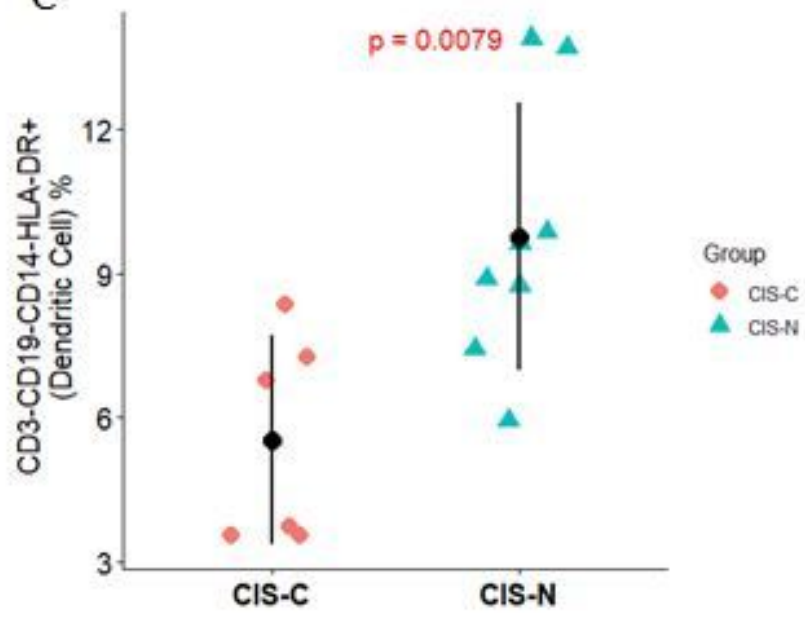

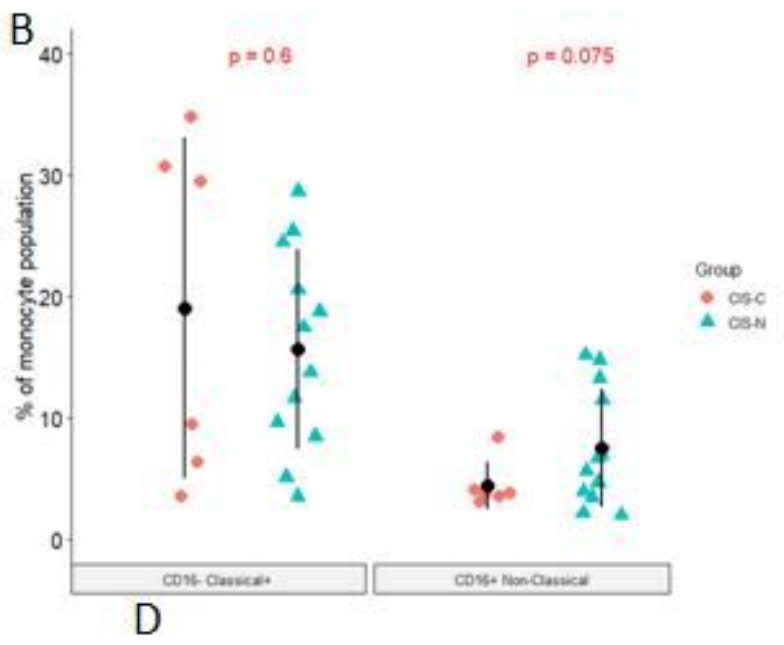

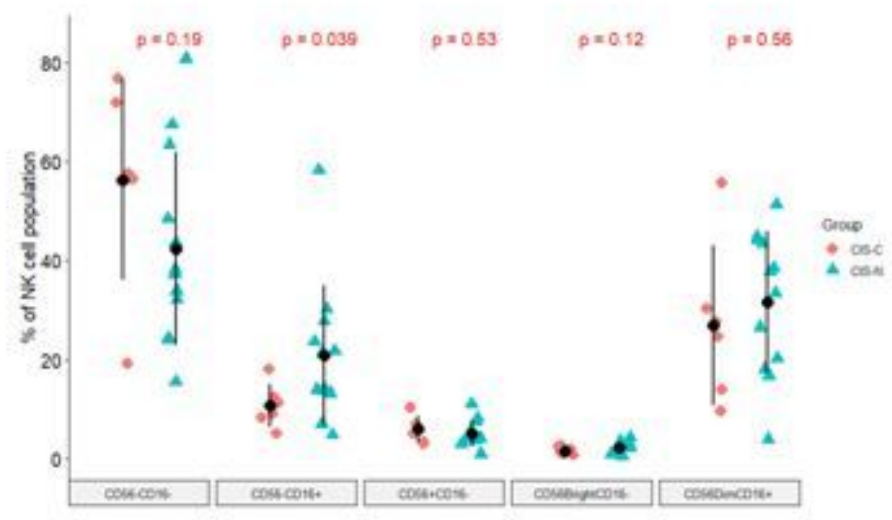

\section{Figure 3}

Monocyte, dendritic cell, NK cell population and their subsets Flow cytometry analysis of fresh peripheral blood samples of patients who converted to MS (CIS-C, $n=7)$ and those who did not (CIS-N, n=12) examining the abundance of monocytes (A), monocyte subsets (B), dendritic cells (C), and NK cell subsets (D). The frequency of cell populations are as a \% of their parent population as indicated, with monocytes and dendritic cells being as a \% of CD3-CD19-cells. The black dot indicates the mean of each group with the vertical line being the SD. The p-values of the comparisons are as shown in the insets. 


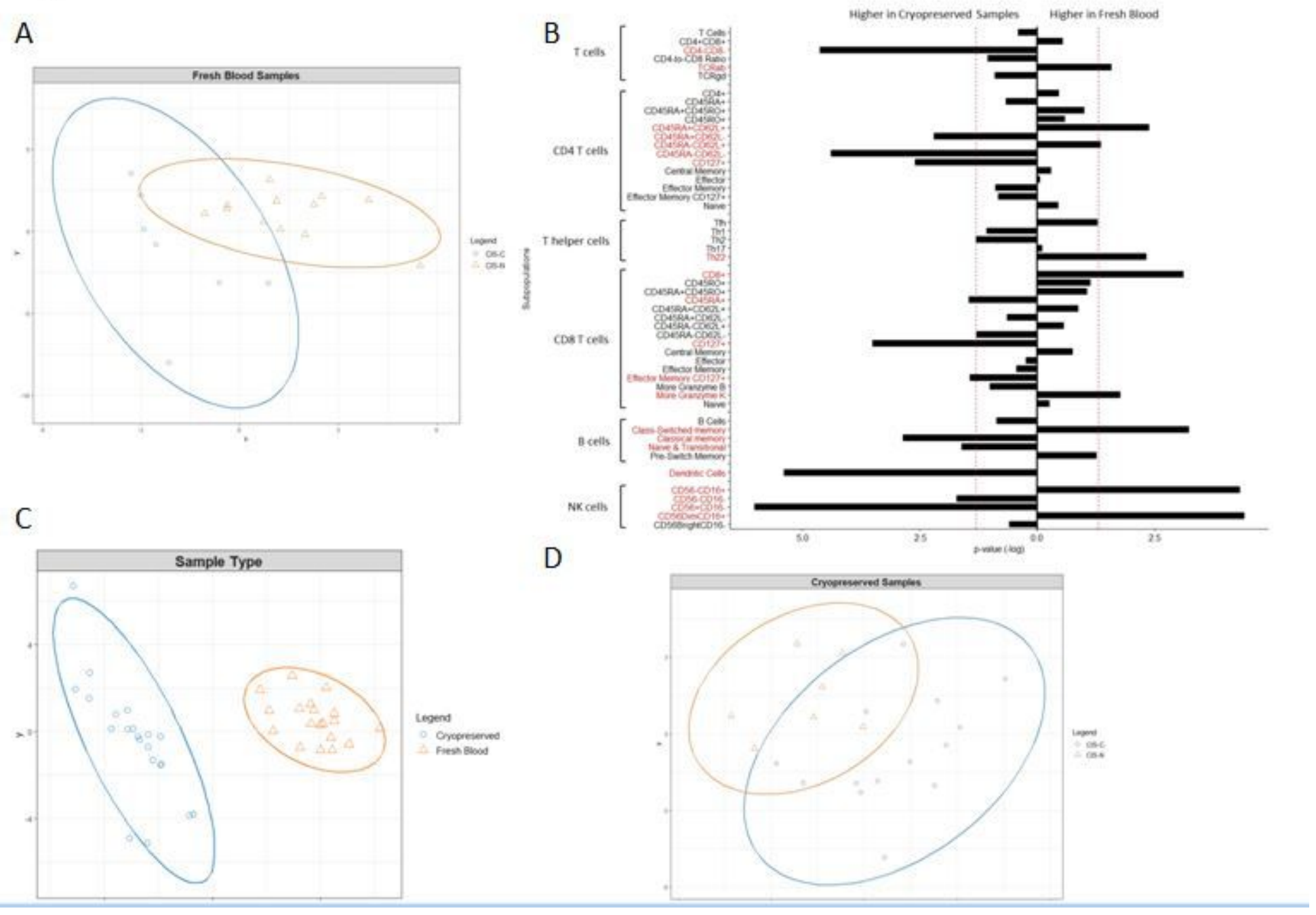

Figure 4

Overall group comparisons of immunophenotypes and comparisons of fresh and cryopreserved samples Comparisons of overall immunophenotype between CIS-C and CIS-N using partial least square discriminant analysis in both fresh blood samples (A), cryopreserved samples (D), and a comparison between the two sample types (C). (B) Comparison of individual cell subpopulations between the two with the results shown as $-\log 10$ transformed $p$ values. The direction of the bars shows whether the cell type is higher in cryopreserved or fresh blood samples. The threshold value of $p=0.05$ is shown with red lines, subpopulations that reach significance are highlighted in red. 